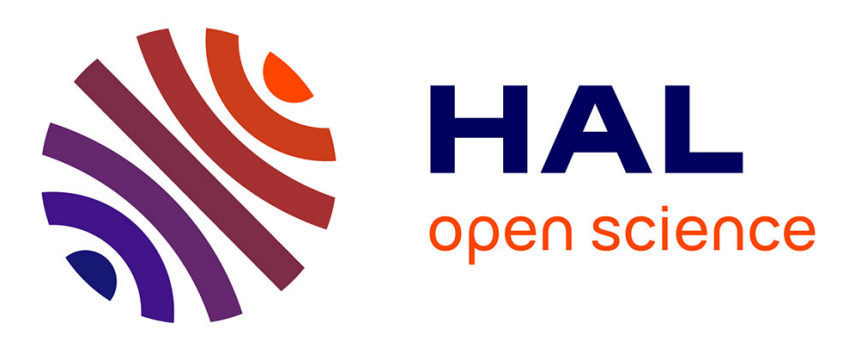

\title{
On the stability of colocated clustered finite volume simplicial discretizations for the 2D Stokes problem
}

Robert Eymard, Raphaele Herbin, Jean-Claude Latché, Bruno Piar

\section{To cite this version:}

Robert Eymard, Raphaele Herbin, Jean-Claude Latché, Bruno Piar. On the stability of colocated clustered finite volume simplicial discretizations for the 2D Stokes problem. Calcolo, 2007, 44 (4), pp.219-234. 10.1007/s10092-007-0138-8 . hal-00136127

\section{HAL Id: hal-00136127 \\ https://hal.science/hal-00136127}

Submitted on 12 Mar 2007

HAL is a multi-disciplinary open access archive for the deposit and dissemination of scientific research documents, whether they are published or not. The documents may come from teaching and research institutions in France or abroad, or from public or private research centers.
L'archive ouverte pluridisciplinaire HAL, est destinée au dépôt et à la diffusion de documents scientifiques de niveau recherche, publiés ou non, émanant des établissements d'enseignement et de recherche français ou étrangers, des laboratoires publics ou privés. 


\title{
On the stability of colocated clustered finite volume simplicial discretizations for the $2 \mathrm{D}$ Stokes problem
}

\author{
R. Eymard ${ }^{1}$, R. Herbin ${ }^{2}$, J.C. Latché ${ }^{3}$, B. Piar ${ }^{3}$ \\ 1 Université de Marne-la-Vallée, France, (robert.eymard@univ-mlv.fr) \\ 2 Université de Provence, France, (herbin@cmi.univ-mrs.fr) \\ 3 Institut de Radioprotection et Sureté Nucléaire (IRSN), France, \\ (jean-claude.latche@irsn.fr, bruno.piar@irsn.fr)
}

August 2006

\begin{abstract}
We study in this paper a novel cell-centered colocated finite volume scheme for the two-dimensional Stokes problem. Its definition involves two grids. The coarsest one is a triangulation of the computational domain in acute angles simplices; these triangles are called clusters. The control volumes grid is a finer one, built by cutting each cluster along the lines joining the mid-edge points to obtain four sub-triangles. By building explicitly a Fortin projection operator, we prove that the pair of discrete spaces associating the classical cell-centered approximation for the velocities and cluster-wide constant pressures is inf-sup stable. In a second step, we prove that a stabilization involving pressure jumps only across the internal edges of the clusters yields a stable scheme with the usual colocated discretization (i.e. with the cell-centered approximation for the velocity and the pressure). We finally give an interpretation of this stabilization as a "minimal stabilization procedure", as introduced by Brezzi and Fortin.
\end{abstract}

\section{Introduction}

The use of colocated cell-centered finite volumes is widespread in Computational Fluid Dynamics, as well in commercial ones (FLUENT, CFX, ...) as in proprietary ones, as developped for instance for nuclear safety problems, which is part of the context of this study. 
Without any regularization procedure, these schemes are known to be unstable when applied to incompressible Navier-Stokes equations; in most developments presented in the literature, this problem is cured by a technique originally proposed by Rhie and Chow [16], the analysis of which, to our knowledge, is still an open problem. Recently, we proposed another stabilization procedure, which can be seen as an extension to finite volumes of the Brezzi-Pitkäranta regularization [4], by now classical in the finite element context. The convergence of this scheme is proven for the steady and unsteady Stokes and Navier-Stokes equations in [8]; for meshes satisfying a particular geometrical assumption, let us say "hypothesis $\left(H_{\mathrm{G}}\right)$ " (see the conclusion for the statement of this assumption), optimal error estimates for the Stokes problem (i.e. first order convergence rate in natural energy norms) are given in [9]. However, for high Reynolds number flows and for meshes reasonable from a computational cost point of view, the amount of stabilization necessary to avoid pressure oscillations was found to severely degrade the accuracy. This led to propose a more local stabilization term, involving pressure jumps only across the internal edges of each cluster of elements. Although being much more general, this approach follows a path similar to the ideas implemented for the Q1-Q0 finite element in $[18,13]$ when going from the so-called "global jump" regularization to the "local jump" one. Some applications of this new scheme are presented in [5], and an analysis for the steady Stokes and Navier-Stokes equations is given in [10]; under the same assumption $\left(H_{\mathrm{G}}\right)$ for the mesh, we again obtain convergence for the steady Stokes and Navier-Stokes equations and optimal error bounds for the Stokes problem, provided some simple geometrical condition is satisfied by the clusters.

However, this analysis based on a direct proof of inf-sup stability estimates is rather intricate and, for particular meshes, a simpler technique based on the classical Fortin lemma [11] is possible; we present in this paper the application of this latter approach to twodimensional simplicial meshes. Clusters are provided by an acute angles triangulation of the computational domain and control volumes are built in a second step by cutting, along the lines joining the midedge points, each triangle of the mesh in four similar triangles. We first prove the stability of the discretization combining the usual cellcentered discretization for the velocity and a cluster-wide constant (i.e. constant by cluster) discretization for the pressure; then we extend this result to a scheme which uses the standard cell-centered discretization also for the pressure and involves a stabilization term involving pressure jumps across the edges internal to the clusters. 
It then follows from the analysis presented in [10] that this scheme is convergent for the Stokes and Navier-Stokes equations, and that first-order error bounds are statisfied for the Stokes problem; as proofs given in [10] apply without modification, these points are not treated here.

The paper is organized as follows. In section 2, we state the continuous problem under consideration. Finite volume approximation spaces are described in section 3 . Then the proposed numerical schemes are presented and their stability is proven in section 4 .

\section{The continuous problem}

The problem under consideration in this paper is the Stokes problem, with homogeneous Dirichlet boundary conditions, which reads:

$$
\begin{aligned}
& \text { Find }(\bar{u}, \bar{p}) \in \mathrm{H}_{0}^{1}(\Omega)^{2} \times \mathrm{L}^{2}(\Omega) \text { with } \int_{\Omega} \bar{p}=0 \text { and such that: } \\
& \begin{array}{ll}
\int_{\Omega} \nabla \bar{u}: \nabla \bar{v}-\int_{\Omega} \bar{p} \operatorname{div} \bar{v}=\int_{\Omega} f \cdot \bar{v} & \forall \bar{v} \in \mathrm{H}_{0}^{1}(\Omega)^{2} \\
\int_{\Omega} \bar{q} \operatorname{div} \bar{u}=0 & \forall \bar{q} \in \mathrm{L}^{2}(\Omega)
\end{array}
\end{aligned}
$$

where $\Omega$ is a polygonal open bounded connected subset of $\mathbb{R}^{2}$ and the left hand side $f \in \mathrm{L}^{2}(\Omega)^{2}$.

The existence and uniqueness of the solution of (1) is a classical result (see e.g. [2], [12] or [1]).

\section{Spatial discretization and discrete functional analysis}

\subsection{Meshing of $\Omega$}

We suppose given a partition of $\Omega$ in triangles having all their inner angles acute; each triangle will be called a cluster, and the set of clusters will be denoted by $\mathcal{G}$. The control volumes are obtained by cutting along the lines joining the mid-edge points each cluster in four similar triangles (see figure 1); the set of control volumes is denoted by $\mathcal{M}$, and, for each control volume $K, G_{K}$ stands for the unique element of $\mathcal{G}$ such that $K \subset G_{K}$.

For each $K$ of $\mathcal{M}$, we denote by $x_{K}$ the intersection point of the perpendicular bisector of the edges of $K$, and, for each edge $\sigma$ of $K$, by $d_{K, \sigma}$ the distance between $x_{K}$ and $\sigma$. The set of edges of the 
mesh is denoted by $\mathcal{E}$; it is the union of the set of internal edges $\mathcal{E}_{\text {int }}$ (included in $\Omega$ ) and external ones $\mathcal{E}_{\text {ext }}$ (included in $\partial \Omega$ ). For each control volume $K, \mathcal{E}(K)$ stands for the set of the three edges of $K$, $\mathcal{N}_{K}$ for the set of the neighbouring control volumes of $K, m_{K}$ for the (2-dimensional) measure of $K$, and $h_{K}$ for its diameter. For each internal edge $\sigma \in \mathcal{E}_{\text {int }}$, separating the control volumes $K$ and $L$, we denote by $d_{\sigma}$ the distance between $x_{K}$ and $x_{L}$ (so $\left.d_{\sigma}=d_{K, \sigma}+d_{L, \sigma}\right)$; such an edge is written $\sigma=K \mid L$. The (one-dimensional) measure of any edge $\sigma \in \mathcal{E}$ is denoted by $m_{\sigma}$. For all $K \in \mathcal{M}$ and $\sigma \in \mathcal{E}(K)$, we denote by $n_{K, \sigma}$ the unit vector normal to $\sigma$ outward to $K$. Finally, the set of edges $\sigma$ of $\mathcal{E}_{\text {int }}$ which are internal to a cluster (i.e. not included in the boundary of a cluster) is denoted by $\mathcal{E}_{\text {int,c }}$.

We shall measure the regularity of the mesh by the parameter $\theta_{\mathcal{M}}$ defined by:

$$
\begin{aligned}
\theta_{\mathcal{M}}=\inf \left\{\frac{m_{\sigma}}{h_{K}}, \frac{d_{K, \sigma}}{h_{K}}, K \in \mathcal{M}, \sigma \in \mathcal{E}(K)\right\} \\
\cup\left\{\frac{h_{K}}{h_{L}}, K \in \mathcal{M}, L \in \mathcal{N}_{K}\right\}
\end{aligned}
$$

Such a mesh is depicted on figure 1 .

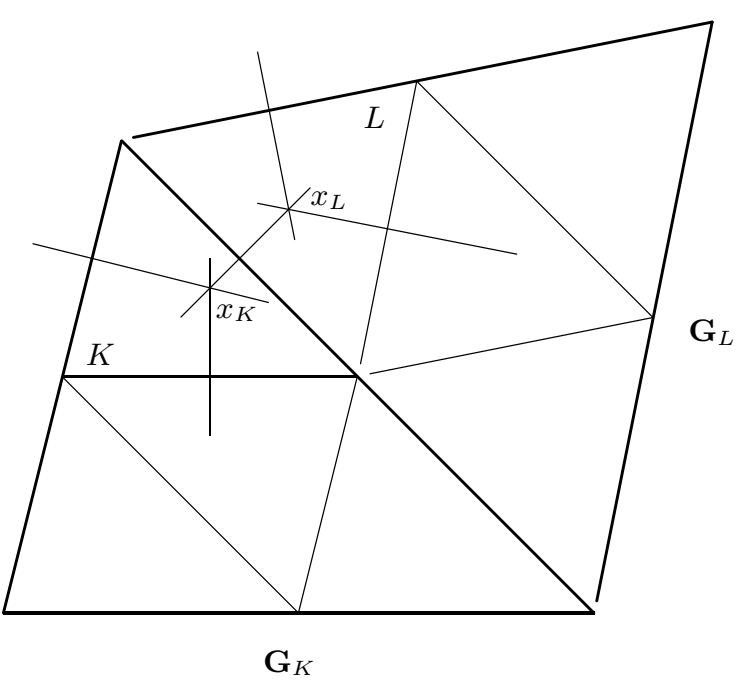

Fig. 1. Exemple of clustered simplicial mesh. 
Finally, we denote by $h_{\mathcal{M}}$ the maximum diameter of the control volumes.

\subsection{Discretization spaces}

Let $\mathrm{H}_{\mathcal{M}}(\Omega) \subset \mathrm{L}^{2}(\Omega)$ be the space of functions which are piecewise constant over each control volume $K \in \mathcal{M}$. For all $w \in \mathrm{H}_{\mathcal{M}}(\Omega)$ and for all $K \in \mathcal{M}$, we denote by $w_{K}$ the constant value of $w$ in $K$. For $(v, w) \in \mathrm{H}_{\mathcal{M}}(\Omega)^{2}$, we define an inner product, which is the discrete analogue of the canonical $\mathrm{H}_{0}^{1}(\Omega)$ bilinear form:

$$
[v, w]_{\mathcal{M}}=\sum_{\substack{\sigma \in \mathcal{E}_{\text {int }}, \sigma=K \mid L}} \frac{m_{\sigma}}{d_{\sigma}}\left(v_{L}-v_{K}\right)\left(w_{L}-w_{K}\right)+\sum_{\substack{\sigma \in \mathcal{E}_{\text {ext }}, \sigma \in \sigma(K)}} \frac{m_{\sigma}}{d_{K, \sigma}} v_{K} w_{K}
$$

Next, we define a norm in $\mathrm{H}_{\mathcal{M}}(\Omega)$ (thanks to the discrete Poincaré inequality (4) given below) by:

$$
\|w\|_{\mathcal{M}}=\left([w, w]_{\mathcal{M}}\right)^{1 / 2} .
$$

These definitions naturally extend to vector-valued functions as follows. For $u=\left(u^{(i)}\right)_{i=1,2} \in \mathrm{H}_{\mathcal{M}}(\Omega)^{2}$ and $v=\left(v^{(i)}\right)_{i=1,2} \in \mathrm{H}_{\mathcal{M}}(\Omega)^{2}$, we define:

$$
\|u\|_{\mathcal{M}}=\left(\sum_{i=1}^{2}\left[u^{(i)}, u^{(i)}\right]_{\mathcal{M}}\right)^{1 / 2} \quad[u, v]_{\mathcal{M}}=\sum_{i=1}^{2}\left[u^{(i)}, v^{(i)}\right]_{\mathcal{M}}
$$

The discrete Poincaré inequality [6, Lemma 9.1 p. 765] reads:

$$
\|w\|_{\mathrm{L}^{2}(\Omega)} \leq \operatorname{diam}(\Omega)\|w\|_{\mathcal{M}}, \quad \forall w \in \mathrm{H}_{\mathcal{M}}(\Omega)
$$

Finally, we denote by $\mathrm{H}_{\mathcal{G}}(\Omega) \subset \mathrm{L}^{2}(\Omega)$ the space of functions which are piecewise constant over each cluster.

\section{Numerical schemes}

\subsection{General formulation}

Finite volume schemes are classically presented as discrete balance equations with a suitable approximation of the fluxes, see e.g. [6]. However, in recent works dealing with cell centered finite volume methods for elliptic problems [7-9], an equivalent variational formulation in adequate functional spaces is introduced, and this presentation is probably more convenient for the analysis of the schemes, as 
the involved variational identities are on the natural path to derive stability estimates. Here we follow this latter approach.

We begin by defining a discrete divergence operator $\operatorname{div}_{\mathcal{M}}$, the expression of which is the same as in [9], and which maps $\mathrm{H}_{\mathcal{M}}(\Omega)^{2}$ onto $\mathrm{H}_{\mathcal{M}}(\Omega)$ and reads:

$$
\begin{aligned}
& \operatorname{div}_{\mathcal{M}} u(x)=\frac{1}{m_{K}} \sum_{\substack{\sigma \in \mathcal{E}_{\text {int }}, \sigma=K \mid L}} m_{\sigma} \frac{d_{L, \sigma} u_{K}+d_{K, \sigma} u_{L}}{d_{\sigma}} \cdot n_{K, \sigma}, \\
& \text { for a.e. } x \in K, \forall K \in \mathcal{M}
\end{aligned}
$$

The adjoint of this discrete divergence defines a discrete gradient $\nabla_{\mathcal{M}}$, mapping $\mathrm{H}_{\mathcal{M}}(\Omega)$ onto $\mathrm{H}_{\mathcal{M}}(\Omega)^{2}$, which takes the expression:

$$
\begin{aligned}
& \nabla_{\mathcal{M}} p(x)=\frac{1}{m_{K}} \sum_{\substack{\sigma \in \mathcal{E}_{\text {int }}, \sigma=K \mid L}} m_{\sigma} \frac{d_{L, \sigma}}{d_{\sigma}}\left(p_{L}-p_{K}\right) n_{K, \sigma}, \\
& \text { for a.e. } x \in K, \forall K \in \mathcal{M}
\end{aligned}
$$

We define the discrete solution as the pair of functions $(u, p)$ solution to the following discrete variational problem:

$$
\begin{aligned}
& \text { Find }(u, p) \in \mathrm{H}_{\mathcal{M}}(\Omega)^{2} \times M \text { with } \int_{\Omega} p=0 \text { and such that: } \\
& \begin{array}{ll}
{[u, v]_{\mathcal{M}}-\int_{\Omega} p \operatorname{div}_{\mathcal{M}} v=\int_{\Omega} f \cdot v} & \forall v \in \mathrm{H}_{\mathcal{M}}(\Omega)^{2} \\
\int_{\Omega} q \operatorname{div}_{\mathcal{M}} u+\langle p, q\rangle_{\lambda, \mathcal{M}}=0 & \forall q \in M
\end{array}
\end{aligned}
$$

where the bilinear form $\langle\cdot, \cdot\rangle_{\lambda, \mathcal{M}}$ defined on $\mathrm{H}_{\mathcal{M}}(\Omega) \times \mathrm{H}_{\mathcal{M}}(\Omega)$ corresponds to a "cluster-wide" stabilization, defined as follows:

$$
\langle p, q\rangle_{\lambda, \mathcal{M}}=\lambda \sum_{\substack{\sigma \in \mathcal{E}_{\text {int,c }}, \sigma=K \mid L}} m_{\sigma}\left(h_{K}+h_{L}\right)\left(p_{L}-p_{K}\right)\left(q_{L}-q_{K}\right)
$$

$\lambda$ being a strictly positive parameter $(\lambda>0)$. This bilinear form is associated to the following semi-norm:

$$
|p|_{\lambda, \mathcal{M}}=\langle p, p\rangle_{\lambda, \mathcal{M}}^{1 / 2}
$$

We will study two choices for the approximation space for the pressure: first, $M=\mathrm{H}_{\mathcal{G}}(\Omega)$ and, second, $M=\mathrm{H}_{\mathcal{M}}(\Omega)$, the first one being analysed in section 4.2 , the second one in section 4.3 . Note that, with the first choice, the stabilization bilinear form vanishes, and we recover the classical (i.e. without stabilization term) setting of a discrete saddle-point problem. 
Choosing $v=\left(1_{K}, 1_{K}\right)^{t}$ (respectively $q=1_{K}$ ) in the first (resp. second) relation of $(7)$, where $1_{K}$ is the characteristic function of the control-volume $K$, yields a discrete momentum (resp. mass) balance equation over the control volume $K$, of the classical finite volume form.

\subsection{Cluster-wide constant pressures}

Our aim in this section is to prove that the discretization combining the usual cell-centered discretization for the velocity and a clusterwide constant discretization for the pressure is stable. To this purpose, we begin by stating a "non-conforming version" of the so-called Fortin lemma, then we build the associated projection operator. The stability of the discretization is an easy consequence of the existence of such an operator.

Lemma 1. We suppose that there exists a continuous projection operator $\Pi_{\mathcal{M}}$ from $\mathrm{H}_{0}^{1}(\Omega)^{2}$ into $\mathrm{H}_{\mathcal{M}}(\Omega)^{2}$ with a continuity constant only depending on $\Omega$ and $\theta_{\mathcal{M}}$ and such that, for any function $u \in \mathrm{H}_{0}^{1}(\Omega)^{2}$ and any cluster $G \in \mathcal{G}$, we have:

$$
\int_{G} \operatorname{div}_{\mathcal{M}}\left(\Pi_{\mathcal{M}} u\right)=\int_{G} \operatorname{div} u
$$

Then the so-called discrete inf-sup condition holds:

$\exists \beta>0$ depending only on $\Omega$ and $\theta_{\mathcal{M}}$ such that,

$$
\forall p \in \mathrm{H}_{\mathcal{G}}(\Omega), \quad \sup _{v \in \mathrm{H}_{\mathcal{M}}(\Omega)^{2}} \frac{\int_{\Omega} p \operatorname{div}_{\mathcal{M}} v}{\|v\|_{\mathcal{M}}} \geq \beta\|p\|_{\mathrm{L}^{2}(\Omega)}
$$

Proof. Let $p \in \mathrm{H}_{\mathcal{G}}(\Omega)$ be given. The fact that the inf-sup condition holds for continuous spaces is wellknown (see e.g. $[14,12,2,1]$ ) and so there exists $\beta_{\mathrm{c}}$ independent of $p$ and $\bar{v} \in \mathrm{H}_{0}^{1}(\Omega)^{2}$ such that:

$$
\frac{\int_{\Omega} p \operatorname{div} \bar{v}}{\|\bar{v}\|_{\mathrm{H}^{1}(\Omega)^{2}}} \geq \beta_{\mathrm{c}}\|p\|_{\mathrm{L}^{2}(\Omega)}
$$

Since $p$ is constant over each cluster, we get from (9):

$$
\int_{\Omega} p \operatorname{div}_{\mathcal{M}}\left(\Pi_{\mathcal{M}} \bar{v}\right)=\int_{\Omega} p \operatorname{div} \bar{v}
$$


On the other hand, by the continuity of the projection operator $\Pi_{\mathcal{M}}$, the function $\Pi_{\mathcal{M}} \bar{v}$ satisfies:

$$
\left\|\Pi_{\mathcal{M}} \bar{v}\right\|_{\mathcal{M}} \leq c_{\Pi_{\mathcal{M}}}\|\bar{v}\|_{\mathrm{H}^{1}(\Omega)^{2}}
$$

From the three previous relations, we thus obtain:

$$
\frac{\int_{\Omega} p \operatorname{div}_{\mathcal{M}}\left(\Pi_{\mathcal{M}} \bar{v}\right)}{\left\|\Pi_{\mathcal{M}} \bar{v}\right\|_{\mathcal{M}}} \geq \frac{\beta_{\mathrm{c}}}{c_{\Pi_{\mathcal{M}}}}\|p\|_{\mathrm{L}^{2}(\Omega)}
$$

We are now going to explicitly build a projection operator suitable for the discretization at hand. To this purpose, for a given cluster $G \in \mathcal{G}$, we will use the local notations defined in figure 2 .

Theorem 1. Let $G$ be a cluster of $\mathcal{G}$ and $\sigma_{i}, i=1,2,3$ its three edges. Let $\bar{u}$ be a function of $\mathrm{H}_{0}^{1}(\Omega)$. We denote by $\bar{u}_{\sigma_{i}}$ the following quantity:

$$
\bar{u}_{\sigma_{i}}=\frac{1}{m_{\sigma_{i}}} \int_{\sigma_{i}} \bar{u}
$$

Then the four following equations define a discrete field $u$ in $G$ (i.e. the restriction to $G$ of a function of $\mathrm{H}_{\mathcal{M}}(\Omega)$ ):

$$
\mid \begin{aligned}
& u_{K_{1}}+u_{K_{2}}=2 \bar{u}_{\sigma_{3}} \\
& u_{K_{2}}+u_{K_{3}}=2 \bar{u}_{\sigma_{1}} \\
& u_{K_{3}}+u_{K_{1}}=2 \bar{u}_{\sigma_{2}} \\
& u_{K_{4}}=\frac{1}{3}\left(u_{K_{1}}+u_{K_{2}}+u_{K_{3}}\right)
\end{aligned}
$$

Repeating this operation for each cluster $G \in \mathcal{G}$ and for each component, we obtain a projection operator mapping $\mathrm{H}_{0}^{1}(\Omega)^{2}$ onto $\mathrm{H}_{\mathcal{M}}(\Omega)^{2}$ which satisfies the assumptions of lemma 1.

Proof. First, we begin by checking that the system (11) admits an unique solution, which reads:

$$
\mid \begin{aligned}
& u_{K_{1}}=\bar{u}_{\sigma_{2}}+\bar{u}_{\sigma_{3}}-\bar{u}_{\sigma_{1}} \\
& u_{K_{2}}=\bar{u}_{\sigma_{1}}+\bar{u}_{\sigma_{3}}-\bar{u}_{\sigma_{2}} \\
& u_{K_{3}}=\bar{u}_{\sigma_{1}}+\bar{u}_{\sigma_{2}}-\bar{u}_{\sigma_{3}} \\
& u_{K_{4}}=\frac{1}{3}\left(\bar{u}_{\sigma_{1}}+\bar{u}_{\sigma_{2}}+\bar{u}_{\sigma_{3}}\right)
\end{aligned}
$$

Second, we need to prove that relation (9) holds, for any function $\left(u^{(1)}, u^{(2)}\right) \in \mathrm{H}_{0}^{1}(\Omega)^{2}$. To this purpose, we check a stronger property, 


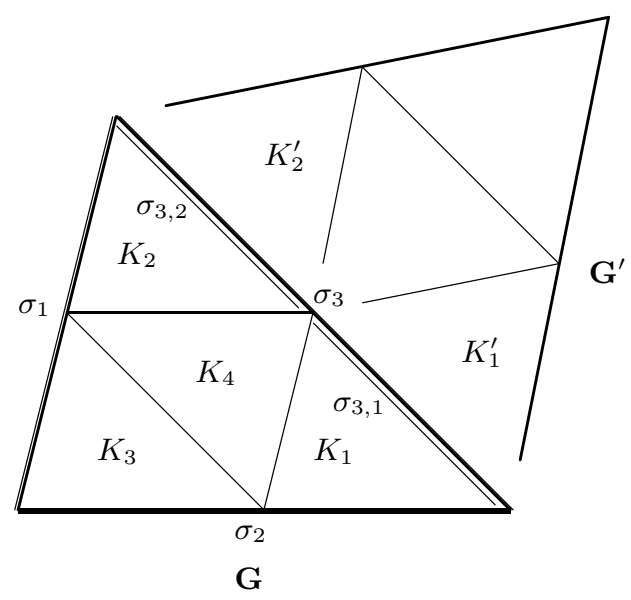

Fig. 2. Local numbering, relative to a given cluster, used in the definition of the Fortin projection operator.

namely that the integral of each component across an edge of a cluster is the same as the integral of the "edge-value" of its projection, evaluated by the interpolation formula of the discrete divergence operator.

Let us consider for instance $\sigma_{3}$ in figure 2. It is easy to observe that the two pairs of triangles sharing respectively $\sigma_{3,1}$ and $\sigma_{3,2}$ are similar. Consequently, the interpolation relations giving in $\operatorname{div}_{\mathcal{M}}$ the velocity on $\sigma_{3,1}$ and $\sigma_{3,2}$, noted $u_{\sigma_{3,1}}$ and $u_{\sigma_{3,2}}$, share the same coefficients, say $\alpha$ and $1-\alpha$, and we get, with the notations of figure 2 :

$$
\begin{aligned}
m_{\sigma_{3,1}} u_{\sigma_{3,1}} & +m_{\sigma_{3,2}} u_{\sigma_{3,2}}=\frac{m_{\sigma_{3}}}{2}\left[u_{\sigma_{3,1}}+u_{\sigma_{3,2}}\right] \\
& =\frac{m_{\sigma_{3}}}{2}\left[\alpha u_{K_{1}}+(1-\alpha) u_{K_{1}}^{\prime}+\alpha u_{K_{2}}+(1-\alpha) u_{K_{2}^{\prime}}\right] \\
& =\frac{m_{\sigma_{3}}}{2}\left[\alpha\left(u_{K_{1}}+u_{K_{2}}\right)+(1-\alpha)\left(u_{K_{1}^{\prime}}+u_{K_{2}^{\prime}}\right)\right] \\
& =m_{\sigma_{3}} \bar{u}_{\sigma_{3}}=\int_{\sigma_{3}} \bar{u}
\end{aligned}
$$

Finally, we have to check the continuity of the projection, i.e. that there exists a positive real number $c_{\text {cont }}$ depending only on $\theta_{\mathcal{M}}$ such that, for each function $\bar{u} \in \mathrm{H}_{0}^{1}(\Omega)$ :

$$
\|u\|_{\mathcal{M}} \leq c_{\text {cont }}|\bar{u}|_{\mathrm{H}_{0}^{1}(\Omega)}
$$


and the continuity of the projection operator for an abitrary velocity field in $\mathrm{H}_{0}^{1}(\Omega)^{2}$ will follow by summation over the two components.

From the expression of the $u_{i}, i=1, \ldots, 4$ given by (12), we see that each difference $u_{K}-u_{L}$, where $K$ and $L$ are two neighbouring control volumes of the mesh, can be expressed as a sum of a bounded number of differences between the quantities $\bar{u}_{\sigma_{1}}, i=1, \ldots, 3$ and their counterparts associated to the neighbouring clusters of $G$. Noticing that, whenever the cluster edge $\sigma$ is included in $\partial \Omega$, we have $\bar{u}_{\sigma}=0$, this property extends to the quantities $u_{K}-0$ appearing in the $\|\cdot\|_{\mathcal{M}}$ norm when one edge of $K$ is included in $\partial \Omega$.

Let us denote by $\varphi_{i}, i=1,2,3$ the basis functions of the finite element $\mathbf{P}_{1}$ discretization over the central triangle $K_{4}, \varphi_{i}$ being equal to 1 at the vertice located on $\sigma_{i}$ and 0 at the other vertices. We define a projection operator $\Pi$ from $\mathrm{H}^{1}\left(K_{4}\right)$ onto the space of linear polynomials by:

$$
\Pi \bar{u}(x)=\tilde{u}(x)=\sum_{i=1,3} \bar{u}_{\sigma_{i}} \varphi_{i}(x)
$$

The projection $\Pi$ is nothing more than a particular Scott and Zhang interpolant [17], the $\mathrm{H}^{1}$ stability of which is known. However, in order to make the presentation self-contained and as the arguments in the case under consideration are simpler than in the general case, we are going to prove the following result:

$$
\|\nabla \tilde{u}\|_{\mathrm{L}^{2}\left(K_{4}\right)} \leq c_{\mathrm{Sz}}\|\nabla \bar{u}\|_{\mathrm{L}^{2}(G)}
$$

where $c_{\mathrm{sz}}$ is a constant depending only on $\theta_{\mathcal{M}}$.

First, we notice that the gradient of each basis function $\varphi_{i}$ is a constant vector and it is easy to check that:

$$
\left\|\nabla \varphi_{i}\right\| \leq \frac{1}{\rho_{K_{4}}} \quad i=1,2,3
$$

where $\rho_{K_{4}}$ stands for the diameter of the largest ball included in $K_{4}$. Consequently, we have:

$$
\|\nabla \tilde{u}\|_{\mathrm{L}^{2}\left(K_{4}\right)} \leq \frac{m_{K_{4}}^{1 / 2}}{\rho_{K_{4}}} \sum_{i=1,3}\left|\bar{u}_{\sigma_{i}}\right|
$$

One then observes that the Scott and Zhang interpolant leaves the constant functions unchanged; we thus have:

$$
\begin{aligned}
\|\nabla \tilde{u}\|_{\mathrm{L}^{2}\left(K_{4}\right)} & =\left\|\nabla\left(\tilde{u}-\frac{1}{m_{G}} \int_{G} \bar{u}\right)\right\|_{\mathrm{L}^{2}\left(K_{4}\right)} \\
& =\left\|\nabla \Pi\left(\bar{u}-\frac{1}{m_{G}} \int_{G} \bar{u}\right)\right\|_{\mathrm{L}^{2}\left(K_{4}\right)}
\end{aligned}
$$


Without loss of generality, we may thus suppose that the function $\bar{u}$ has a zero mean value over $G$. By the Cauchy-Schwarz inequality, we have:

$$
\left|\bar{u}_{\sigma_{i}}\right| \leq \frac{1}{m_{\sigma_{i}}^{1 / 2}}\|\bar{u}\|_{\mathrm{L}^{2}\left(\sigma_{i}\right)}
$$

and thus, by a trace inequality which can be found in [19]:

$$
\left|\bar{u}_{\sigma_{i}}\right| \leq \frac{1}{m_{\sigma_{i}}^{1 / 2}}\left[2 \frac{m_{\sigma_{i}}}{m_{G}}\right]^{1 / 2}\left[\|\bar{u}\|_{\mathrm{L}^{2}(G)}+h_{G}\|\nabla \bar{u}\|_{\mathrm{L}^{2}(G)}\right]
$$

The Poincaré inequality (see [15] for a value of the Poincaré constant for zero mean valued functions valid for any convex domain) thus yields:

$$
\left|\bar{u}_{\sigma_{i}}\right| \leq c\left[\frac{1}{m_{G}}\right]^{1 / 2} h_{G}\|\nabla \bar{u}\|_{L^{2}(G)}
$$

where $c$ is a constant real number and, finally, gathering inequalities (15) and (16):

$$
\|\nabla \tilde{u}\|_{\mathrm{L}^{2}\left(K_{4}\right)} \leq 3 c\left[\frac{m_{K_{4}}}{m_{G}}\right]^{1 / 2} \frac{h_{G}}{\rho_{K_{4}}}\|\nabla \bar{u}\|_{\mathrm{L}^{2}(G)}
$$

which implies the bound (14).

We now return to the stability of $\Pi_{\mathcal{M}}$. Let $a_{i}$ be the middle of the $\sigma_{i}$ of $G$. Each groupment $\left(\bar{u}_{\sigma_{i}}-\bar{u}_{\sigma_{j}}\right)^{2}$ can then be estimated as follows:

$$
\left(\bar{u}_{\sigma_{i}}-\bar{u}_{\sigma_{j}}\right)^{2} \leq\left(m_{\left[a_{i}, a_{j}\right]}\right)^{2}\|\nabla \Pi \bar{u}\|^{2}=\frac{\left(m_{\left[a_{i}, a_{j}\right]}\right)^{2}}{m_{K_{4}}}\|\nabla \tilde{u}\|_{L^{2}\left(K_{4}\right)}^{2}
$$

and thus:

$$
\left(\bar{u}_{\sigma_{i}}-\bar{u}_{\sigma_{j}}\right)^{2}=c \frac{\left(m_{\left[a_{i}, a_{j}\right]}\right)^{2}}{m_{K_{4}}}\|\nabla \bar{u}\|_{\mathrm{L}^{2}(G)}^{2}
$$

Remarking that the quantity $\|\nabla \bar{u}\|_{\mathrm{L}^{2}(G)}^{2}$ will appear a bounded number of times in the summation giving $\|u\|_{\mathcal{M}}$, this estimates implies that the bound (13) holds, which concludes the proof.

We are now in position to state the stability of the scheme. To this purpose, we define the bilinear form $B_{\mathcal{M}}(\cdot, \cdot ; \cdot, \cdot)$ by:

$$
B_{\mathcal{M}}(u, p ; v, q)=[u, v]_{\mathcal{M}}-\int_{\Omega} p \operatorname{div}_{\mathcal{M}} v+\int_{\Omega} q \operatorname{div}_{\mathcal{M}} u
$$


where $u, v$ and $p, q$ stand for respectively two elements of $\mathrm{H}_{\mathcal{M}}(\Omega)^{2}$ and two elements of $\mathrm{H}_{\mathcal{G}}(\Omega)$. The numerical scheme under consideration (7) with cluster-wide constant pressures can be written equivalently:

Find $(u, p) \in \mathrm{H}_{\mathcal{M}}(\Omega)^{2} \times \mathrm{H}_{\mathcal{G}}(\Omega)$ such that:

$$
B_{\mathcal{M}}(u, p ; v, q)=\int_{\Omega} f \cdot v \quad \forall(v, q) \in \mathrm{H}_{\mathcal{M}}(\Omega)^{2} \times \mathrm{H}_{\mathcal{G}}(\Omega)
$$

Theorem 2 (Stability of the scheme). For each pair $u \in \mathrm{H}_{\mathcal{M}}(\Omega)^{2}$ and $p \in \mathrm{H}_{\mathcal{G}}(\Omega)$ (i.e. such that the pressure $p$ is constant over each cluster), there exists $\tilde{u} \in \mathrm{H}_{\mathcal{M}}(\Omega)^{2}$ and $\tilde{p} \in \mathrm{H}_{\mathcal{G}}(\Omega)$ such that:

$$
\|\tilde{u}\|_{\mathcal{M}}+\|\tilde{p}\|_{\mathrm{L}^{2}(\Omega)} \leq c_{1}\left[\|u\|_{\mathcal{M}}+\|p\|_{\mathrm{L}^{2}(\Omega)}\right]
$$

and:

$$
\|u\|_{\mathcal{M}}^{2}+\|p\|_{L^{2}(\Omega)}^{2} \leq c_{2} B_{\mathcal{M}}(u, p ; \tilde{u}, \tilde{p})
$$

where $c_{1}$ and $c_{2}$ are two positive real numbers depending only on $\Omega$ and $\theta_{\mathcal{M}}$.

Proof. Let $u \in \mathrm{H}_{\mathcal{M}}(\Omega)^{2}$ and $p \in \mathrm{H}_{\mathcal{G}}(\Omega)$ be given. First, we note that:

$$
B_{\mathcal{M}}(u, p ; u, p)=\|u\|_{\mathcal{M}}^{2}
$$

Then, by (10), we know that there exists $v \in \mathrm{H}_{\mathcal{M}}(\Omega)^{2}$ such that:

$$
\mid \begin{aligned}
& \|v\|_{\mathcal{M}}=\|p\|_{L^{2}(\Omega)} \\
& -\int_{\Omega} p \operatorname{div}_{\mathcal{M}} v \geq \beta\|p\|_{L^{2}(\Omega)}^{2}
\end{aligned}
$$

where $\beta>0$ only depends on $\Omega$ and $\theta_{\mathcal{M}}$. We thus have, by the Cauchy-Schwarz and Young inequalities:

$$
\begin{aligned}
B_{\mathcal{M}}(u, p ; v, 0) & =-\int_{\Omega} p \operatorname{div}_{\mathcal{M}} v+[u, v]_{\mathcal{M}} \\
& \geq \beta\|p\|_{L^{2}(\Omega)}^{2}-\left[\frac{\beta}{2}\|p\|_{L^{2}(\Omega)}^{2}+\frac{1}{2 \beta}\|u\|_{\mathcal{M}}^{2}\right] \\
& =\frac{\beta}{2}\|p\|_{L^{2}(\Omega)}^{2}-\frac{1}{2 \beta}\|u\|_{\mathcal{M}}^{2}
\end{aligned}
$$

Then, by bilinearity of $B_{\mathcal{M}}(\cdot, \cdot ; \cdot, \cdot)$, we see that both (17) and (18) hold with $\tilde{u}=u+\beta v$ and $\tilde{p}=p$. 


\subsection{Clustered triangles discretizations}

We now turn to the analysis of the scheme with an approximation for the pressure which is constant over each control volume rather than constant over each cluster. Consequently, the stabilization terms involving the pressure jump through the interior edges of the clusters do not vanish anymore.

We begin by a technical lemma, which can be seen as a very specific formulation of a discrete "cluster by cluster" Poincaré inequality.

Lemma 2. Let $p \in \mathrm{H}_{\mathcal{M}}(\Omega)$ a function which vanishes over the inner triangle (i.e. the triangle denoted $K_{4}$ on figure 2) of each cluster. Then the following bound holds:

$$
\|p\|_{\mathrm{L}^{2}(\Omega)}^{2} \leq \frac{1}{2 \lambda}|p|_{\mathcal{M}, \lambda}^{2}
$$

Proof. Let $G$ be a cluster of $\mathcal{G}$. Using a local numbering of the unknowns, we have by assumption $p_{4}=0$ and:

$$
\begin{aligned}
& \|p\|_{L^{2}(G)}^{2}=\sum_{i=1}^{3} m_{K_{i}} p_{i}^{2}=\sum_{i=1}^{3} m_{K_{i}}\left(p_{i}-p_{4}\right)^{2} \\
& \quad \leq \max _{i=1,2,3}\left[\frac{m_{K_{i}}}{m_{K_{i} \mid K_{4}}\left(h_{K_{i}}+h_{K_{4}}\right)}\right] \sum_{i=1}^{3} m_{K_{i} \mid K_{4}}\left(h_{K_{i}}+h_{K_{4}}\right)\left(p_{i}-p_{4}\right)^{2}
\end{aligned}
$$

Thanks to geometrical considerations, the first factor of the above right-hand-side is smaller than $1 / 2$. The result then follows by summation over the clusters.

As in the previous section, we define a bilinear form $B_{\mathcal{M}}(\cdot, \cdot ; \cdot, \cdot)$ which is now given by:

$$
B_{\mathcal{M}}(u, p ; v, q)=[u, v]_{\mathcal{M}}-\int_{\Omega} p \operatorname{div}_{\mathcal{M}} v+\int_{\Omega} q \operatorname{div}_{\mathcal{M}} u+\langle p, q\rangle_{\lambda, \mathcal{M}}
$$

where $u, v$ and $p, q$ stand respectively for two elements of $\mathrm{H}_{\mathcal{M}}(\Omega)^{2}$ and two elements of $\mathrm{H}_{\mathcal{M}}(\Omega)$. Once again, the scheme under consideration reads:

Find $(u, p) \in \mathrm{H}_{\mathcal{M}}(\Omega)^{2} \times \mathrm{H}_{\mathcal{M}}(\Omega)$ such that:

$$
B_{\mathcal{M}}(u, p ; v, q)=\int_{\Omega} f \cdot v \quad \forall(v, p) \in \mathrm{H}_{\mathcal{M}}(\Omega)^{2} \times \mathrm{H}_{\mathcal{M}}(\Omega)
$$

We then have the following stability result. 
Theorem 3 (Stability of the stabilized scheme). For each pair $u \in \mathrm{H}_{\mathcal{M}}(\Omega)^{2}$ and $p \in \mathrm{H}_{\mathcal{M}}(\Omega)$, there exists $\tilde{u} \in \mathrm{H}_{\mathcal{M}}(\Omega)^{2}$ and $\tilde{p} \in$ $\mathrm{H}_{\mathcal{M}}(\Omega)$ such that:

$$
\|\tilde{u}\|_{\mathcal{M}}+\|\tilde{p}\|_{\mathrm{L}^{2}(\Omega)} \leq c_{1}\left[\|u\|_{\mathcal{M}}+\|p\|_{\mathrm{L}^{2}(\Omega)}\right]
$$

and:

$$
\|u\|_{\mathcal{M}}^{2}+\|p\|_{L^{2}(\Omega)}^{2} \leq c_{2} B_{\mathcal{M}}(u, p ; \tilde{u}, \tilde{p})
$$

where $c_{1}$ and $c_{2}$ are two positive real numbers depending only on $\lambda$, $\Omega$ and $\theta_{\mathcal{M}}$.

Proof. The proof follows basically the same lines as for cluster-wide constant pressures. Let $u \in \mathrm{H}_{\mathcal{M}}(\Omega)^{2}$ and $p \in \mathrm{H}_{\mathcal{M}}(\Omega)$ be given. First, we note that:

$$
B_{\mathcal{M}}(u, p ; u, p)=\|u\|_{\mathcal{M}}^{2}+|p|_{\mathcal{M}, \lambda}^{2}
$$

Then, we define $\hat{p}$ the function of $\mathrm{H}_{\mathcal{G}}(O)$ (i.e. constant over each cluster) which is equal to $p$ over the inner triangle of each cluster. By (10), we know that there exists $v \in \mathrm{H}_{\mathcal{M}}(\Omega)^{2}$ such that:

$$
\mid \begin{aligned}
& \|v\|_{\mathcal{M}}=\|\hat{p}\|_{L^{2}(\Omega)} \\
& -\int_{\Omega} \hat{p} \operatorname{div}_{\mathcal{M}} v \geq \beta\|\hat{p}\|_{L^{2}(\Omega)}^{2}
\end{aligned}
$$

where $\beta>0$ only depends on $\Omega$ and $\theta_{\mathcal{M}}$. We thus have, by Young's inequality, lemma 2 applied to the difference $p-\hat{p}$ and the stability in $\mathrm{H}_{\mathcal{M}}(\Omega)^{2}$ of the discrete divergence operator:

$$
\begin{aligned}
& B_{\mathcal{M}}(u, p ; v, 0)= {[u, v]_{\mathcal{M}}-\int_{\Omega}(p-\hat{p}) \operatorname{div}_{\mathcal{M}} v-\int_{\Omega} \hat{p} \operatorname{div}_{\mathcal{M}} v } \\
& \geq \beta\|\hat{p}\|_{\mathrm{L}^{2}(\Omega)}^{2}-\left[\frac{\beta}{4}\|\hat{p}\|_{\mathrm{L}^{2}(\Omega)}^{2}+\frac{1}{\beta}\|u\|_{\mathcal{M}}^{2}\right] \\
& \quad \quad-\left[\frac{\beta}{4}\|\hat{p}\|_{\mathrm{L}^{2}(\Omega)}^{2}+\frac{c_{\mathrm{div}}^{2}}{\beta}\|p-\hat{p}\|_{\mathrm{L}^{2}(\Omega)}^{2}\right] \\
&=\frac{\beta}{2}\|\hat{p}\|_{\mathrm{L}^{2}(\Omega)}^{2}-\frac{1}{\beta}\|u\|_{\mathcal{M}}^{2}-\frac{c_{\text {div }}^{2}}{2 \beta \lambda}|p|_{\mathcal{M}, \lambda}^{2}
\end{aligned}
$$

where $c_{\text {div }}$ is the continuity constant of $\operatorname{div}_{\mathcal{M}}$ and, to obtain the last term, we remark that $|\hat{p}|_{\mathcal{M}, \lambda}=0$. Let $\alpha$ be given by:

$$
\alpha=\beta \min \left(\frac{1}{2}, \frac{\lambda}{c_{\mathrm{div}}^{2}}\right)
$$

Then, by bilinearity of $B_{\mathcal{M}}(\cdot, \cdot ; \cdot, \cdot)$, we get:

$$
B_{\mathcal{M}}(u, p ; u+\alpha v, p) \geq \frac{1}{2}\|u\|_{\mathcal{M}}^{2}+\frac{1}{2}|p|_{\mathcal{M}, \lambda}^{2}+\frac{\alpha \beta}{2}\|\hat{p}\|_{L^{2}(\Omega)}^{2}
$$


To conclude, we remark that:

$$
|p|_{\mathcal{M}, \lambda}=|p-\hat{p}|_{\mathcal{M}, \lambda} \geq \lambda\|p-\hat{p}\|_{\mathrm{L}^{2}(\Omega)}
$$

and, by the triangular inequality, we see that both (17) and (18) hold with $\tilde{u}=u+\alpha v$ and $\tilde{p}=p$.

Remark 1. The concept of "minimal stabilization procedure" was introduced for mixed finite element methods by Brezzi and Fortin in [3]. Applied to the two dimensional Stokes problem, a particular consequence of the abstract result proven in their paper reads as follows. Let $M \subset \mathrm{L}^{2}(\Omega)$ be an approximation space for the pressure such that the pair $(V, M)$ is inf-sup stable, where $V \subset \mathrm{H}_{0}^{1}(\Omega)^{2}$ stands for a discretisation space for the velocity. Let $P \subset \mathrm{L}^{2}(\Omega)$ be another approximation space such that $M \subset P$. Then the Stokes problem approximated with the pair $(V, P)$ may be stabilized by the term:

$$
\lambda \int_{\Omega}[p-\Pi p][q-\Pi q]
$$

where $\Pi$ is a projection operator from $P$ onto $M$.

Let us evaluate this stabilization term, choosing $\mathrm{H}_{\mathcal{M}}(\Omega)$ for $P$, $\mathrm{H}_{\mathcal{G}}(\Omega)$ for $M$ and, for $\Pi$, the projection from $\mathrm{H}_{\mathcal{M}}(\Omega)$ onto $\mathrm{H}_{\mathcal{G}}(\Omega)$ defined, for $p \in \mathrm{H}_{\mathcal{M}}(\Omega)$, by setting the value of $\Pi p$ over the cluster to the value of $p$ in the central control volume (i.e. the control volume denoted by $K_{4}$ on figure 2). Then we have, for any cluster $G$ :

$$
\begin{aligned}
\lambda \int_{G}[p-\Pi p][q-\Pi q] & \lambda \sum_{\sigma=K_{i} \mid K_{4}, i=1,2,3} m_{K_{i}}\left(p_{K_{i}}-p_{K_{4}}\right)\left(q_{K_{i}}-q_{K_{4}}\right)
\end{aligned}
$$

which is similar to the stabilization used here, with $m_{K \mid L}\left(h_{K}+h_{L}\right)$ replaced by the equivalent quantity $m_{K}$. The scheme presented in this paper can thus be seen as resulting from an extension to the finite volume context of the minimal stabilization procedure.

\section{Conclusion}

We proved in this paper the stability of two finite volume schemes for the two-dimensional Stokes problem based on simplicial meshes. They may be shown to imply the existence and uniqueness of the discrete solution, and to lead to error bounds which, for instance, take the following form for the stabilized scheme [10]. 
Theorem 4 (Error estimate). Let $\lambda \in(0,+\infty)$ be given. We suppose that the solution of the continuous problem $(1),(\bar{u}, \bar{p})$, lies in $\mathrm{H}^{2}(\Omega)^{2} \cap \mathrm{H}_{0}^{1}(\Omega)^{2} \times \mathrm{H}^{1}(\Omega)$. Let $(u, p) \in \mathrm{H}_{\mathcal{M}}(\Omega)^{2} \times \mathrm{H}_{\mathcal{M}}(\Omega)$ be the solution to (7). Then there exists a positive real number $c$ depending only on $\Omega, \theta_{\mathcal{M}}$ and $\lambda$ such that the following inequality holds:

$$
\left\|u-\bar{u}_{\mathcal{M}}\right\|_{\mathcal{M}}+\left\|p-\bar{p}_{\mathcal{M}}\right\|_{\mathrm{L}^{2}(\Omega)} \leq c h_{\mathcal{M}}\left[\|\bar{u}\|_{\mathrm{H}^{2}(\Omega)^{2}}+|\bar{p}|_{\mathrm{H}^{1}(\Omega)}\right]
$$

where $\bar{u}_{\mathcal{M}}$ is the function of $\mathrm{H}_{\mathcal{M}}(\Omega)^{2}$ defined by $\left(\bar{u}_{\mathcal{M}}\right)_{K}=\bar{u}\left(x_{K}\right)$ and $\bar{p}$ is the function of $\mathrm{H}_{\mathcal{M}}$ the value of which on each control volume $K$ is the mean value of $\bar{p}$ over $K$.

For a cluster-wide constant pressure, the result would be the same, relacing the average of $\bar{p}$ on the control volumes by the average on the clusters. The proof of this result can be found in [10] and is not repeated here. It makes use of consistency estimates also given in [10]. For the divergence term, this bound relies on the fact that, for each pair of neighbouring control volumes, the segment $\left[x_{K}, x_{L}\right]$ crosses the edge $K \mid L$ at its mass center; this is the assumption called $\left(H_{\mathrm{G}}\right)$ in the introduction of this paper. This hypothesis holds for the construction of the point $x_{K}$ used here; note that this geometrical property, unfortunately, is not verified anymore for tetrahedra in the three-dimensional case, which should make an extension of the stability analysis presented here to 3D problems of little interest in practice. However, a similar theory holds for rectangular and orthogonal parallelepipeds.

\section{References}

1. F. Boyer and P. Fabrie. Eléments d'analyse pour l'étude de quelques modèles d'écoulements de fluides visqueux incompressibles, volume 52 of Mathématiques et Applications. Springer-Verlag, 2006.

2. F. Brezzi and M. Fortin. Mixed and Hybrid Finite Element Methods. Springer series in Computational Mathematics. Springer-Verlag, 1991.

3. F. Brezzi and M. Fortin. A minimal stabilisation procedure for mixed finite element methods. Numerische Mathematik, 89:457-491, 2001.

4. F. Brezzi and J. Pitkäranta. On the stabilization of finite element approximations of the Stokes equations. In W. Hackbusch, editor, Efficient Solution of Elliptic Systems, volume 10 of Notes Num.Fluid Mech., pages 11-19. Vieweg, 1984.

5. E. Chénier, R. Eymard, and O. Touazi. Numerical results using a colocated finite volume scheme on unstructured grids for incompressible fluid flows. Numerical Heat Tranfer Part B: Fundamentals, 49(3):259-276, 2006.

6. R. Eymard, T Gallouët, and R. Herbin. Finite volume methods. volume VII of Handbook of Numerical Analysis, pages 713-1020. North Holland, 2000. 
7. R. Eymard, T. Gallouët, and R. Herbin. A finite volume scheme for anisotropic diffusion problems. C. R. Math. Acad. Sci. Paris, 339(4):299302, 2004.

8. R. Eymard, R. Herbin, and J.C. Latché. Convergence analysis of a colocated finite volume scheme for the incompressible Navier-Stokes equations on general 2 or 3D meshes. SIAM Journal on Numerical Analysis. in press.

9. R. Eymard, R. Herbin, and J.C. Latché. On a stabilized colocated finite volume scheme for the Stokes problem. Mathematical Modelling and Numerical Analysis. in press.

10. R. Eymard, R. Herbin, J.C. Latché, and B. Piar. On colocated clustered finite volume schemes for incompressible flow problems. submitted.

11. M. Fortin. An analysis of the convergence of mixed finite element methods. RAIRO, Anal. Numér., 11:341-354, 1977.

12. V. Girault and P.-A. Raviart. Finite Element Methods for Navier-Stokes Equations. Theory and Algorithms., volume 5 of Springer Series in Computational Mathematics. Springer-Verlag, 1986.

13. N. Kechkar and D. Silvester. Analysis of locally stabilized mixed finite element methods for the Stokes problem. Mathematics of Computation, 58(197):1-10, January 1992.

14. J. Nečas. Equations aux dérivées partielles. Presses de l'Université de Montréal, 1965.

15. L.E. Payne and H.F. Weinberger. An optimal poincaré-inequality for convex domains. Arch. Rational Mech. Anal., 5:286-292, 1960.

16. C.M. Rhie and W.L. Chow. Numerical study of the turbulent flow past an airfoil with trailing edge separation. AIAA Journal, 21(11):1525-1532, 1983.

17. L.R. Scott and S. Zhang. Finite element interpolation of nonsmooth functions satisfying boundary conditions. Mathematics of Computation, 54:483-493, 1990.

18. D.J. Silvester and N. Kechkar. Stabilised bilinear-constant velocity-pressure finite elements for the conjugate gradient solution of the Stokes problem. Computer Methods in Applied Mechanics and Engineering, 79:71-86, 1990.

19. R. Verfürth. Error estimates for some quasi-interpolation operators. Mathematical Modelling and Numerical Analysis, 33(4):695-713, 1999. 